\title{
Beyond the ERPs - Startle Response is Better Outlined by Whole Brain and Spectral EEG Features
}

\author{
Mengting Liu ${ }^{1^{*}}$, Xuemin Wang ${ }^{2}$ \\ ${ }^{1}$ Department of Psychological and Brain Science, University of Delaware, DE, \\ 19716, USA; \\ ${ }^{2}$ Department of Biomedical Engineering, Tianjin University, Tianjin, 300072, \\ China.
}

*Corresponding to: Dr. Mengting Liu, Department of Psychological and Brain Science, University of Delaware, DE, 19716, USA. Email: mliu@psych.udel.edu.

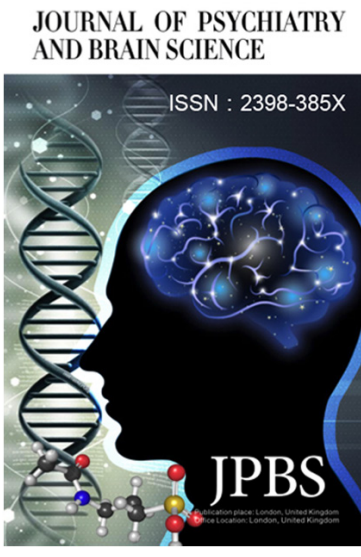

http://jpbs.qingres.com

GOPEN ACCESS

DOI: 10.20900/jpbs.20170009

Received: March 14, 2017

Accepted: May 16, 2017

Published: June 25, 2017

Copyright: ๑2017 Cain et al. This is an open access article distributed under the terms of the Creative Commons Attribution License, which permits unrestricted use, distribution, and reproduction in any medium, provided the original author and source are credited.

\section{ABSTRACT}

Objective: To isolate an spacial and spectral EEG biomarker that is reliable for characterizing amygdala associated startle response.

Methods: A spatial-spectral filter of spectrally weighted Common Spatial Pattern (CSP) analysis was applied to identify spacial and spectral features for EEG startle response. Support vector machine classifier with radial basis kernel was applied to identify the reliability of discovered features.

Results: Theta band in frontocentral and alpha band in bilateral frontal cortex holds the most discriminant components for startle and control. The cross validation using SVM yielded a $80.3 \%$ classification accuracy, which is much higher than just ERPs features (65.5\%).

Conclusion: Amygdala activity may be assessed via EEG spatially in specific frequency bands.

Keywords: EMG startle response; Event-related potentials; Common spatial pattern; Cross validation

\section{INTRODUCTION}

The EMG startle response is considered a defensive reaction to potential threats that manifests as a unique eye blink-like pattern in the EMG. Startle response has been demonstrated to be robustly mediated by amygdala activity. Unfortunately in EEG and other neural 
studies $^{[1]}$ the isolation of amygdala activation is nearly impossible. EMG startle response has been widely applied in indexing emotion, or emotional imagery and attentional engagement studies. As a boost of EMG startle response operation, identifying biomarkers ${ }^{[2]}$ for amygdala activity is becoming increasingly important.

The startle reflex is elicited by presenting loud acoustic noises via headphones or puffs of air to the eyeball ${ }^{[3]}$. Stimulus such as loud acoustic noises doesn't necessary elicit startle reflex due to various of reasons, e.g. habituation, reallocation of attentional resources, and personality trait. One access to distinguish startle occurrence is via eye blink amplitudes. Startle response is supposed to occur if eye blink EMG peak amplitude reaches three times standard deviation above EMG baseline. Startle trials do not reach this criterion, they were usually treated as non-response startle response.

Currently, event-related potentials (ERPs) is the most prominent approach to characterize startle response and amydgala activity in EEG studies. Startle response releases several brain waves in EPRs. Specifically, the N100, which is the negative deflection of ERP that occurs around $100 \mathrm{~ms}$ after the onset of startle probe, and the P300, which is the positive deflections of ERP that occurs around $300 \mathrm{~ms}$ after the onset of startle probe, were mostly studied ${ }^{[4]}$. N100 was thought to index the early sensory processing ${ }^{[5]}$ and $\mathrm{P} 300$ is hypothesized to index attentional and initial memory storage events ${ }^{[6]}$.

N100 and P300 waves are devalued for their limited roles in charactering startle reflex. Especially, spatial ${ }^{[7]}$ and spectral ${ }^{[8]}$ information are lost when they serve as biomarkers for startle reflex. Common spatial pattern (CSP) ${ }^{[9]}$ is a mathematical procedure used in signal processing for separating a multivariate signal into additive subcomponents which have maximum differences in variance between two groups of data. CSP was further developed by adding simultaneous spatiotemporal filter optimization algorithm incorporates non-homogeneous weighting of the cross-spectrum matrices, which is called spectral weighted CSP (sw-CSP) ${ }^{[10]}$. By applying sw-CSP, a combination of spatial and spectral features that are able to maximally differentiating response-startle trials and non-response trials are disclosed.

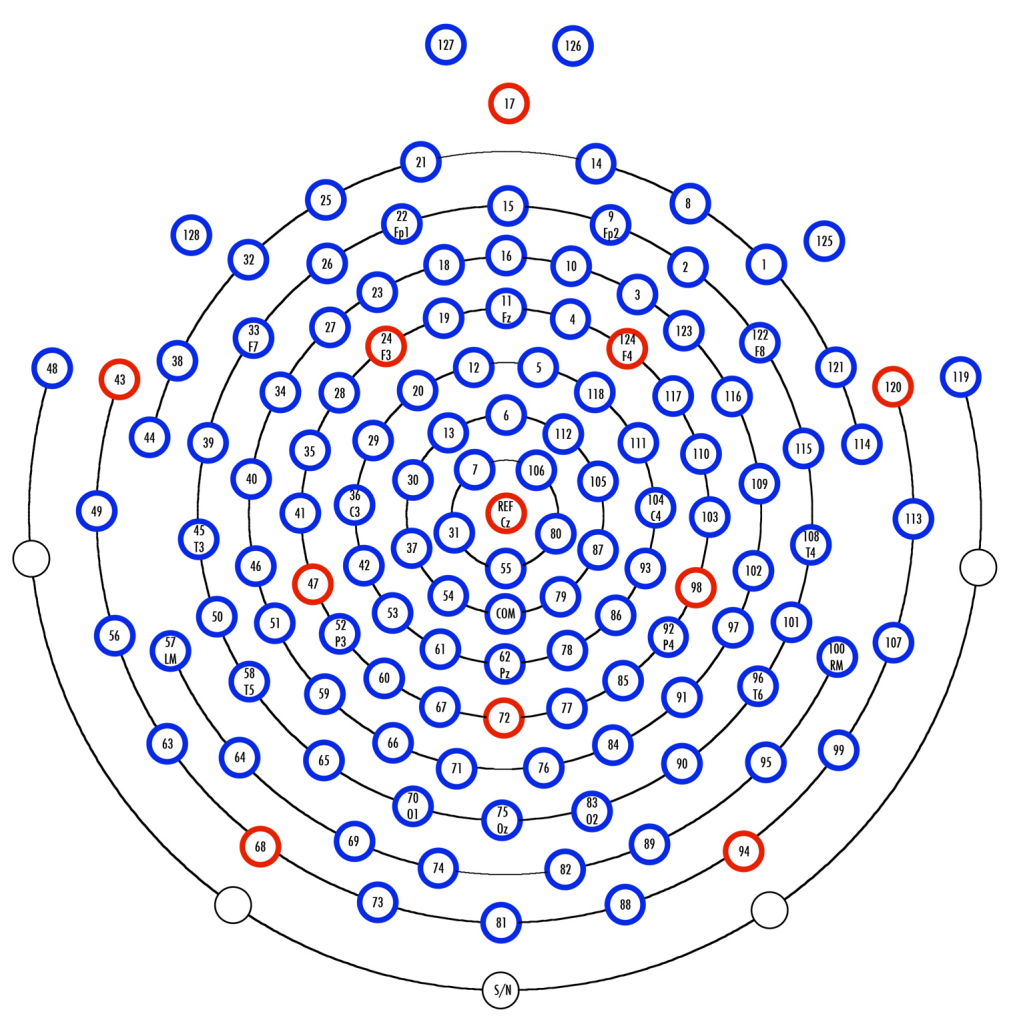

Fig. 1 The channel map as observed from the top of the subject's head with the front of the head pointing upward. 


\section{METHODS}

\subsection{Participants and Procedure}

Sixty-five participants from Tianjin University (33 Males) completed the study. EEG signals were recorded using 128 channels HydroGel Geodesic Sensor Net (Fig. 1) with the Net-Station 5.3 software [11]. Two electrodes were placed next to each other $1 \mathrm{~cm}$ below the right eye to record startle eye-blink responses. All signals were amplified and antialiasing low-pass filtered at $100 \mathrm{~Hz}$. The data was then digitized at a sample rate of $256 \mathrm{~Hz}$.

\subsection{Startle Acquisition and Extraction}

To elicit startle responses, a $40 \mathrm{~ms}$ burst of white noise $(100 \mathrm{db})$ was presented (Bose Quietcomfort 25). Startle responses were obtained from electromyographic (EMG) recordings of the right orbicularis occuli muscle using two EMG electrodes. As with the EEG data, startle activity was digitized at a sample rate of $256 \mathrm{~Hz}$. Eye blink EMG data was then filtered by a $28 \mathrm{~Hz}$ high-pass FIR filter and then rectified. Peaks appearing between $20 \mathrm{~ms}$ and 150 ms after administration of the acoustic startle probe were operationalized as startle responses ${ }^{[12]}$.

\subsection{EEG Preprocessing}

Startle EEG signal was epoched and with stimulus locked from $500 \mathrm{~ms}$ pre-stimulus presentation to $1000 \mathrm{~ms}$ post-stimulus. EEG artifacts were removed via FASTER (Fully Automated Statistical Thresholding for EEG artifact Rejection) ${ }^{[13]}$, an automated approach to cleaning EEG data that is based on multiple iterations of independent component and statistical thresholding analyses. In FASTER, EEG trials are filtered by a $0.3 \mathrm{~Hz}$ high pass FIR filter, and baseline corrected using the time series from $100 \mathrm{~ms}$ preceding the onset of the tasks. All artifact components are identified and removed as a z-score for that variable, e.g., independent components are identified as eye blink components via their correlation with simultaneous EOG channels and are removed from the EEG signal.

\subsection{ERP Measure}

The P300 was scored as the average activity at $\mathrm{CPz}$ (where it was maximal) between $320-360 \mathrm{~ms}$, and the $\mathrm{N} 100$ was scored as the average activity at $\mathrm{FCz}$ (where it was maximal) between $100-140 \mathrm{~ms}$.

\subsection{Validation Using Classification}

A classifier using support vector machine (SVM) with radial basis kernel (RBF) was applied to identify how the selected features are able to differentiate the response-startle reflex and non-response startle reflex. The cross validation in testing data was compared between using N100 and P300 features and spacial-spectral features.

\section{RESULTS}

\subsection{ERPs Comparison}

The P300 was evident at centroparietal sites and was maximal approximately $320 \mathrm{~ms}$ after the startle probe (see Fig. 2, right). Univariate ANOVA represented a main effect of condition, $F(1,820)$ $=8.892, p=0.003$, indicating that startle trials have larger $\mathrm{P} 300$ magnitude $(\mathrm{M}=5.64, \mathrm{SD}=0.51)$ compared to control trials $(M=3.47, S D=0.51)$. The N100 was evident at frontocentral sites and was maximal approximately $110 \mathrm{~ms}$ after probe presentation (see Fig. 2, left). Univariate ANOVA suggested a main effect of condition, $F(1,820)=$ $35.26, p<0.001$, indicating that startle trials have larger N100 magnitude $(M=4.25, S D=0.40)$ compared to control trials $(M=0.88, S D=0.30)$.

Results indicated that response startle trials N100 power and P300 power are larger than non-response trials, as shown in Fig. 2. To further validate the ERPs feature, a SVM with RBF kernel based classifier was conducted on response startle trials and non-response startle trials using N100 and P300 features, result yielded a classification accuracy of $65.5 \%$.

\subsection{Common Spatial Pattern}

A common spatial pattern spatial filter identified significant differentiable patterns in frontocentral and left lateral-central cortex between startle responses and controls. Fig. 3 illustrated how CSP helps differentiate patterns in EEG data. In addition, a spatial-spectral filter of spectrally weighted CSP indicated that the theta band in the two areas holds the most discriminant components (Fig. 4). To further verify, a classifier was applied to the identified spatial and spectral features. The cross validation in testing data yielded a $80.3 \%$ classification accuracy. 

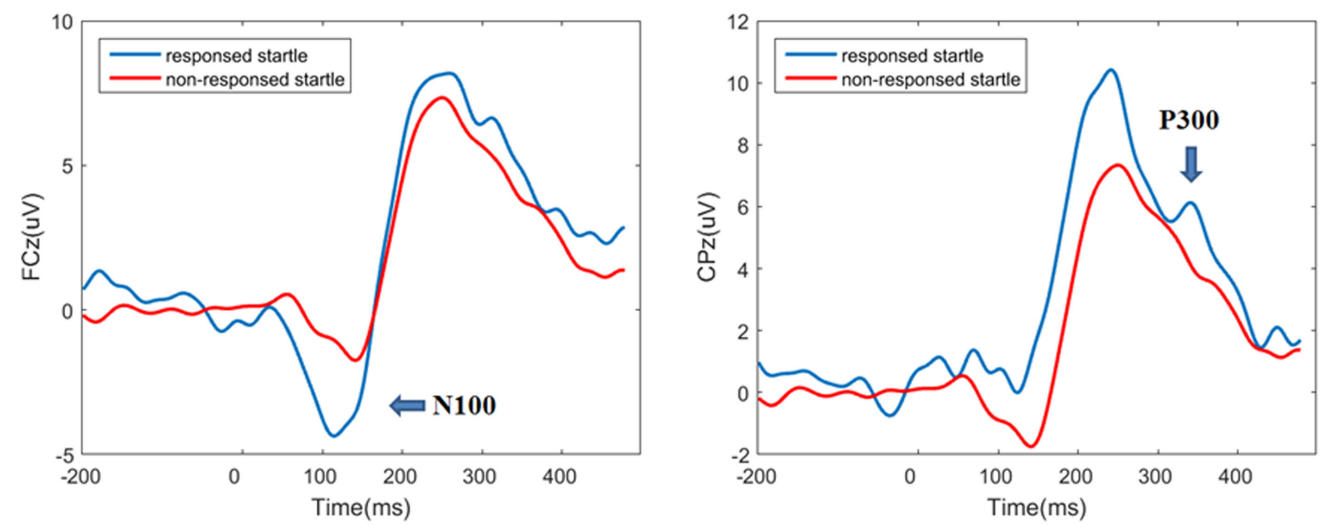

Fig. 2 ERP grand-average waveforms at FCz for response and non-response trials (left), and ERP grandaverage waveforms at $\mathrm{CPz}$ for response and non-response trials (right).
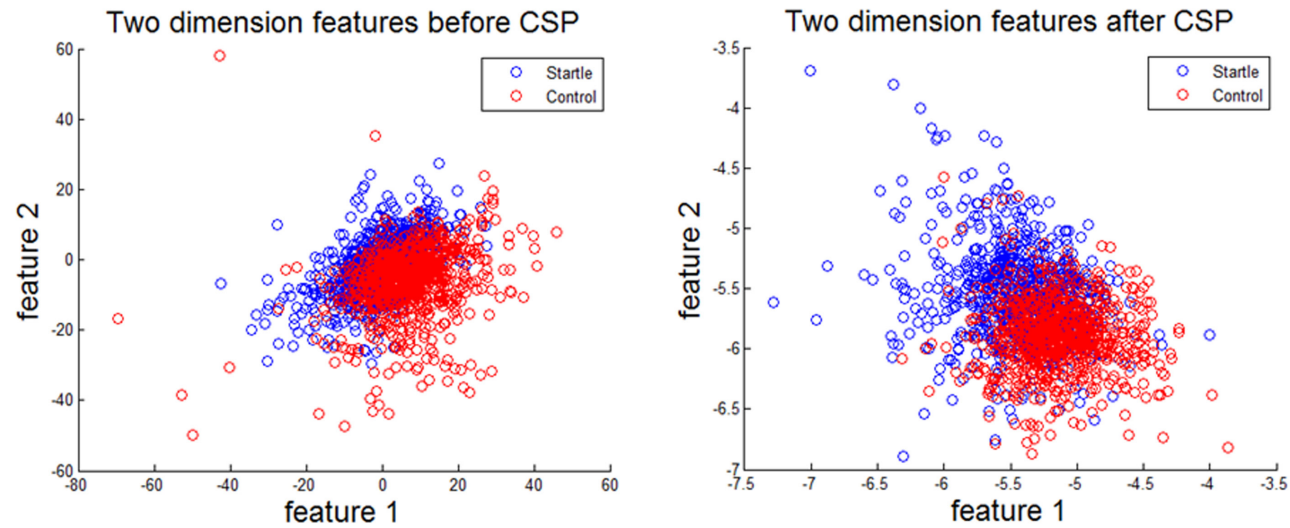

Fig. 3 An illustration of CSP on differentiating EEG patterns between response startle trials and nonresponse trials.
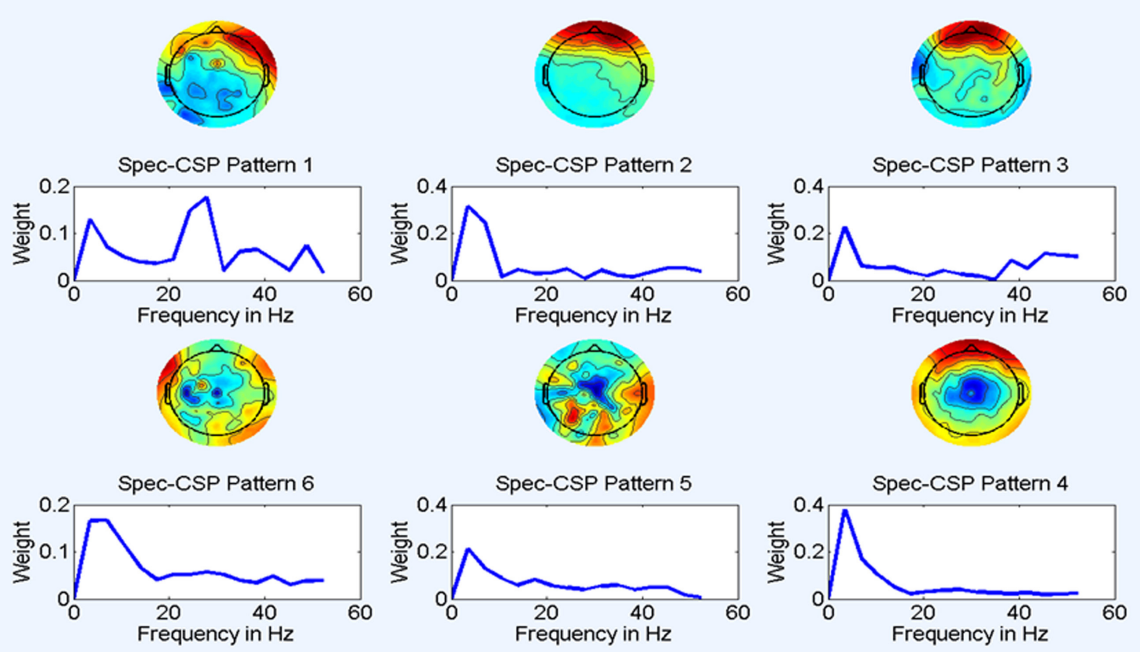

Fig. 4 Theta band in frontocentral and alpha band in bilateral frontal cortex hold the most discriminant components for identifying amygdala activity. 


\section{CONCLUSION}

Startle eye-blink reflex has been utilized as an indicator for the defensive reactions in both human and animal studies. Variation of startle eye-blink patterns in different emotional and attentional contexts have been utilized as an important gauge in identifying the emotion regulation level. Extended research demonstrated that startle reflex is robustly mediated by amygdala activity. Up to now, there is very little research reporting on the interpretation of amygdala activity in EEG data, neither reporting on spatial or spectral components in EEG that contributes towards amygdala activity. As an expansion of EMG startle response operation, identifying an explicit biomarkers for amygdala activity is becoming increasingly important.

Traditional ERP features are not able to represent all aspects of amygdala activity in brain, where spatial and spectral information were omitted. By using spectral weighted common spatial pattern analysis, we isolated more comprehensive neural features for amygdala activity in EEG data. Results indicated that theta band in frontocentral and alpha band in bilateral frontal cortex holds the most discriminant components for identifying amygdala activity.

One limitation of this study is that startle reflex was marked using an eye-blink EMG amplitude. An EMG peak amplitude higher than baseline by three times of standard deviation or more was taken as a response-startle trial, while an EMG peak amplitude higher than baseline by less than three times of standard deviation was a non-response-startle trial. This criterion, although frequently utilized in startle analysis, is vague for its physiological meaning. Results could differ if different criteria was used (e.g., EMG with peak amplitude of about 2 - times of standard deviation above baseline could be a weak startle response for these with stable psychological endurance). Future studies will target at analyzing the psychophysiological meaning of those nonresponse startle trials, especially trials with eye-blink amplitudes only slightly smaller than three times standard deviation of baseline.

\section{ACKNOWLEDGEMENTS}

This work has no funding support.

\section{DECLARATION OF INTERESTS}

The authors declare no conflict of interests.

\section{REFERENCES}

1. Jiang W, Luo J, Nangia S. Multiscale approach to investigate self-assembly of telodendrimer based nanocarriers for anticancer drug delivery. Langmuir. 2015 Apr 14; 31(14): 4270-4280.

2. Mantena V, Jiang WJ, Li J, Mckenzie R. Prostate cancer biomarker identification using MALDIMS data: initial results. Life Science Systems and Applications Workshop. 2009. LiSSA 2009. IEEE / NIH. IEEE, 2009.

3. Lissek S, Baas JM, Pine DS, Orme K, Dvir S, Nugent M, Rosenberger E, Rawson E, Grillon C. Airpuff startle probes: an efficacious and less aversive alternative to white-noise. Biol Psychol. 2005; 68(3): 283-297.

4. Putnam LE, Roth WT. Effects of stimulus repetition, duration, and rise time on startle blink and automatically elicited P300. Psychophysiology. 1990; 27(3): 275-297.

5. Lang PJ, Bradley MM, Cuthbert BN. Emotion, attention, and the startle reflex. Psychol Rev. 1990; 97(3): 377.

6. Polich J. Updating P300: an integrative theory of P3a and P3b. Clin Neurophysiol. 2007; 118(10): 2128-2148.

7. Liu MT, Kuo CC, Chiu AW. Statistical threshold for nonlinear granger causality in motor intention analysis. Conf Proc IEEE Eng Med Biol Soc. 2011; 2011: 5036-5039.

8. Liu M, Kuo CC, Chiu AW. EEG theta rhythm analysis using nonlinear granger causality and approximate decomposition for decoding of motor intention. Conference proceedings:... Annual International Conference of the IEEE Engineering in Medicine and Biology Society. IEEE Engineering in Medicine and Biology Society. Conference. Vol. 2012. 2012.

9. Wang Y, Gao S, Gao X. Common Spatial Pattern Method for Channel Selelction in Motor Imagery Based Brain-computer Interface. Conf Proc IEEE Eng Med Biol Soc. 2005; 5: 5392-5395.

10. Tomioka R, sDornhege G, Nolte G, Blankrtz B, Aihara K, Muller KR. Spectrally weighted common spatial pattern algorithm for single trial EEG classification. Dept. Math. Eng., Univ. Tokyo, Tokyo, Japan, Tech. Rep 40 (2006). 
11. Liu MT, Kuo CC, Chiu AW. Non-linear Granger causality and its frequency decomposition in decoding human upper limb movement intentions. International Journal of Biomedical Engineering and Technology. 2013; 12(1): 1-25.

12. Blumenthal TD, Cuthbert BN, Filion DL, Hackley S, Lipp OV, Boxtel V. Committee report: Guidelines for human startle eyeblink electromyographic studies. Psychophysiology. 2 005; 42(1): 1-15.

13. Nolan $H$, Whelan $R$, Reilly RB. FASTER: fully automated statistical thresholding for EEG artifact rejection. J Neurosci Methods. 2010 Sep 30; 192(1): 152-162. 